http://dx.doi.org/10.1590/1678-4162-7748

Arq. Bras. Med. Vet. Zootec., v.68, n.4, p.945-952, 2016

\title{
Comportamento biomecânico de diferentes placas de avanço da tuberosidade da tíbia em cães: estudo comparativo ex vivo
}

\author{
[Biomechanical behavior of two different tibial tuberosity advancement plates in dogs: \\ comparative ex vivo biomechanical study] \\ K.C.I. Yamauchi ${ }^{1}$, C.R.A. Ferrigno ${ }^{2}$, C.A.M. Pereira ${ }^{3}$, R.A.O. Cavalcanti ${ }^{2}$, \\ J.H.H. Grisi-Filho ${ }^{2}$ \\ ${ }^{1}$ Faculdade de Medicina Veterinária - Universidade de Cuiabá - UNIC - Cuiabá, MT \\ ${ }^{2}$ Faculdade de Medicina Veterinária e Zootecnia - Universidade de São Paulo - FMVZ-USP - São Paulo, SP \\ ${ }^{3}$ Faculdade de Medicina - Universidade de São Paulo - IFM-USP - São Paulo, SP
}

\begin{abstract}
RESUMO
Este é o primeiro estudo que compara o comportamento biomecânico de duas diferentes placas de avanço da tuberosidade tibial (ATT). Com o objetivo de minimizar a incidência de falhas de implante, foram realizados ensaios biomecânicos em 10 pares de membros pélvicos de cadáveres de cães. No membro pélvico direito, foi colocada placa de ATT fixa por garfo, e no membro pélvico esquerdo placa fixa por parafusos. Os ensaios foram realizados utilizando-se máquina universal de ensaios mecânicos Kratos ${ }^{\circledR}$, modelo KE3000, dotada de célula de carga de $3000 \mathrm{~N}$, com velocidade de ensaio de $20 \mathrm{~mm} / \mathrm{min}$. O parâmetro força e os gráficos gerados foram gravados por meio de sistema de aquisição analógica TRACOMP-W95 (TRCV61285). A média da carga máxima até a falha foi de 128,70kg/F (mínimo $104,55 \mathrm{~kg} / \mathrm{F}$ e máximo $151,80 \mathrm{~kg} / \mathrm{F}$ ) e de $141,99 \mathrm{~kg} / \mathrm{F}$ (mínimo $111,60 \mathrm{~kg} / \mathrm{F}$ e máximo $169,65 \mathrm{~kg} / \mathrm{F}$ ) no grupo utilizando garfo e parafusos, respectivamente. $\mathrm{O}$ desvio-padrão dos grupos garfo e parafuso foi baixo, 12,99 e 17,21, respectivamente. Foi encontrada diferença significativa $(\mathrm{P}=0,03)$ entre as médias dos grupos. Com base nos resultados obtidos, conclui-se que existe diferença estatística significativa na resistência promovida entre as placas testadas, quando se observou que a placa fixa por parafusos promove maior resistência.
\end{abstract}

Palavras-chave: ruptura de ligamento cruzado, ensaio biomecânico, resistência, implantes

\begin{abstract}
This is the first study to compare the biomechanical strength between the two different TTA plates. With the purpose of minimizing the incidence of implant failure, biomechanical tests were performed on 10 pairs of cadaveric hind limbs from dogs. The right hind limb of each dog was used as a model for the TTA plate fixed by fork, and the left pelvic limb was used as a model for the TTA plate fixed by screws. Tests were performed using a universal mechanical testing Kratos ${ }^{\circledR}$ machine, model KE 3000, equipped with a load cell of $3000 \mathrm{~N}$ with a test speed of $20 \mathrm{~mm} / \mathrm{min}$. The strength parameter and graphs generated were recorded via analog acquisition system TRACOMP-W95 (TRCV61285). The mean load to failure was $128.70 \mathrm{~kg} / \mathrm{F}$ (minimum $104.55 \mathrm{~kg}$ and maximum $151.80 \mathrm{~kg} / \mathrm{F}$ ) and $141.99 \mathrm{~kg} / \mathrm{F}$ (minimum $111.60 \mathrm{~kg} / \mathrm{F}$ and maximum $169.65 \mathrm{~kg} / \mathrm{F}$ ) in the fork and screws groups respectively. The standard deviation of the fork and screws groups was low: 12.99 and 17.21 respectively. Significant difference $(P=0.03)$ between means of the groups was observed. From the results obtained it was concluded that there is a statistically significant difference in the resistance between plates and the plate fixed by screws promotes increased strength.
\end{abstract}

Keywords: cruciate ligament rupture, biomechanical test, resistance, implants

Recebido em 11 de junho de 2014

Aceito em 7 de agosto de 2015

E-mail: itokelly@yahoo.com.br 


\section{INTRODUÇÃO}

Avanço da tuberosidade tibial (ATT) é uma técnica para correção da RLCCr em cães, desenvolvida por Montavon et al. (2002). O sucesso da técnica de ATT em reduzir ou eliminar as forças de deslocamento cranial da tíbia em joelhos de cães com RLCCr tem sido documentado em estudos experimentais (Apelt et al., 2007; Kim et al., 2008/2009; Guerrero et al., 2009) e em estudos clínicos publicados recentemente (Hoffmann et al., 2006; Lafaver et al., 2007; Voss et al., 2008; Kemper et al., 2011; Steinberg et al., 2011; Wolf et al., 2012).

Uma descrição retrospectiva dos estudos tem relatado bons resultados clínicos baseados na avaliação da satisfação do proprietário (Hoffmann et al., 2006; Lafaver et al., 2007): $83 \%$ dos proprietários relataram um notável aumento ou o retorno completo da função do membro (Hoffmann et al., 2006), e 90\% dos proprietários julgaram os resultados clínicos como bons ou excelentes (Lafaver et al., 2007). A falha do implante é relatada em 1 a $5 \%$ dos membros operados. Esse número foi atribuído a erro de técnica ou resistência do implante, que foi considerado fraco, por isso o implante tem sido modificado (Lafaver et al., 2007).

O presente estudo objetiva avaliar comparativamente, por meio de ensaio biomecânico de tração, ex vivo, o comportamento e a resistência promovida por dois tipos de placas de ATT (placa convencional fixa por garfo e placa fixa por parafusos na região da tuberosidade tibial) após procedimento cirúrgico em tíbias de cães doadores. A hipótese é a de que existe diferença na resistência promovida por cada uma delas quando submetidas à força de tração sobre o ligamento patelar na região da tuberosidade tibial.

\section{MATERIAL E MÉTODO}

Os ensaios biomecânicos foram realizados no Setor de Biomecânica do Laboratório de Ortopedia e Traumatologia Comparada do Departamento de Cirurgia da Faculdade de Medicina Veterinária e Zootecnia da Universidade de São Paulo (FMVZ-USP). O trabalho foi aprovado pela Comissão de Ética no Uso de Animais da FMVZ-USP e protocolado sob o número 1933/2010.
Foram utilizados 10 pares de membros pélvicos de cadáveres de cães que vieram a óbito por razões independentes deste estudo, totalizando 20 peças. Os cães doadores apresentavam diferentes raças, mas tamanho e peso corporal aproximado. As peças foram coletadas imediatamente após a morte dos animais, por desarticulação coxofemoral. Foram realizadas radiografias das tíbias em projeção mediolateral para cálculo do ângulo do platô tibial, conforme metodologia estabelecida por Slocum e Devine (1983). Foram utilizadas somente peças cujas tíbias apresentavam APT até $24^{\circ}$ e compatíveis com placas de ATT convencionais para garfos com cinco pontas ou placas fixas por dois parafusos e espaçador de $9 \mathrm{~mm}$ e livre de afecção ortopédica que comprometesse o estudo. Após remoção de tecidos moles, estas foram armazenadas em freezer convencional, com temperatura de $-20^{\circ} \mathrm{C}$ (Apelt et al., 2007; Etchepareborde et al., 2010; Jung et al., 2011). As peças foram armazenadas em embalagens plásticas com dimensões de $50 \mathrm{~cm}$ de altura e $30 \mathrm{~cm}$ de largura. Em cada embalagem, foi acondicionado um par de membros (fêmur e tíbia direitos e esquerdos do mesmo animal) em sacos plásticos idênticos, transparentes, com identificação de idade, sexo, raça, peso, ângulo do platô tibial do cão proveniente, feita com caneta permanente resistente a água.

Os implantes utilizados neste estudo foram da marca Securos ${ }^{\circledR}$, sendo 10 placas de ATT convencionais (Securos $\AA$, Estados Unidos) para garfos de cinco pontas, 10 placas de ATT fixas por parafusos (X-gen, Securos ${ }^{\circledR}$, Estados Unidos), 10 garfos com cinco pontas (Securos ${ }^{\circledR}$, Estados Unidos) e 20 espaçadores de $9 \mathrm{~mm}$ (Securos ${ }^{\circledR}$, Estados Unidos).

Uma vez congeladas, as peças foram descongeladas somente uma vez para realização da técnica de colocação dos implantes e, em seguida, foram submetidas ao ensaio biomecânico. As peças foram submetidas à colocação dos implantes obedecendo à seguinte regra: tíbias direitas com placas convencionais fixas por garfo e tíbias esquerdas do mesmo doador com placas fixas por parafusos (Fig. 1). A técnica foi realizada por um único cirurgião ortopédico. Para colocação dos implantes na tíbia direita, foi necessário instrumental específico para ATT. A tíbia foi fixa com face medial voltada para cima. Após marcação da osteotomia 
na tuberosidade tibial com bisturi elétrico, foi realizada osteotomia distal parcial dela $(50 \%$ do comprimento total da tuberosidade) em sentido longitudinal. Orifícios na tuberosidade tibial foram realizados com broca $2,0 \mathrm{~mm}$ e guia específico para colocação do garfo de fixação de cinco pontas. A placa foi recalcada e fixa com o garfo. Em seguida, a osteotomia longitudinal da tuberosidade tibial foi concluída com serra oscilatória à bateria e o espaçador $9 \mathrm{~mm}$ foi posicionado e fixo na região proximal da osteotomia por meio de parafusos $2,0 \mathrm{~mm}$. Por fim, a placa foi posicionada e fixa por parafusos $2,7 \mathrm{~mm}$ na região diafisária da tíbia. Todos os parafusos foram colocados obedecendo à regra padrão. A técnica realizada para esse modelo de placa foi a mesma descrita por Montavon et al. (2002).

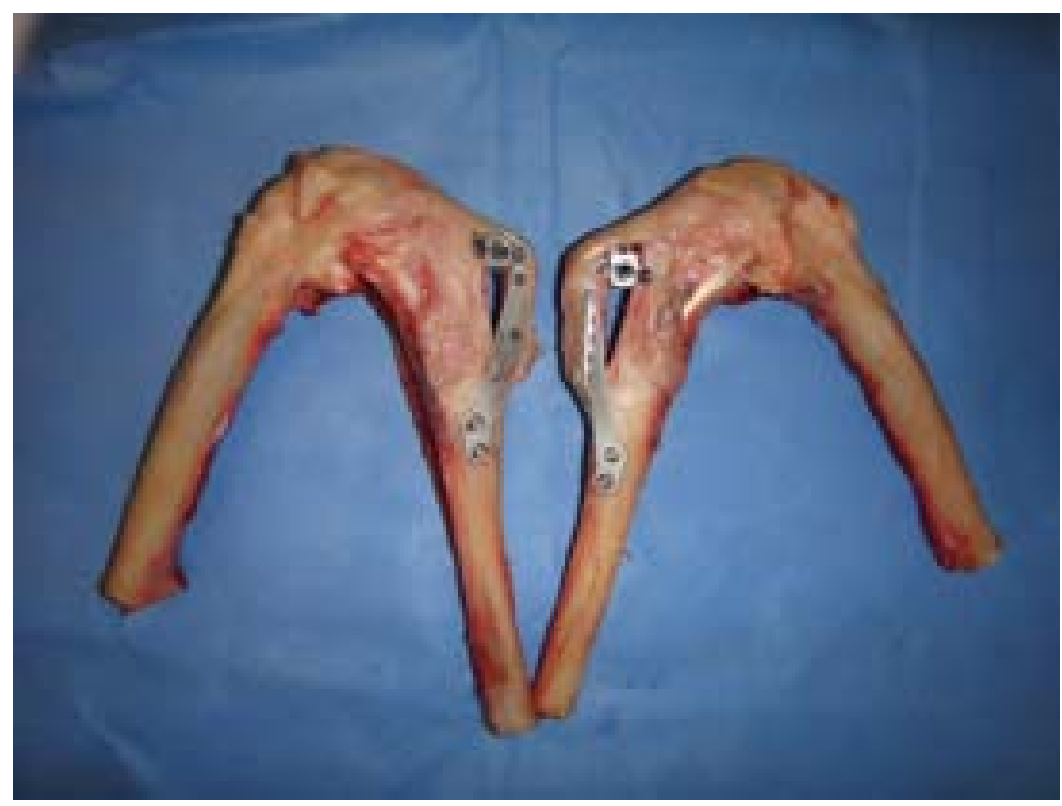

Figura 1. Vista medial dos corpos de prova (teste piloto) a serem submetidos ao teste de tração, os quais consistem em fêmur e tíbia dos membros direito e esquerdo do mesmo cão doador, tendo sido a tíbia direita submetida à técnica de ATT com tuberosidade fixa por placa convencional (garfo) e a tíbia esquerda submetida à mesma técnica com tuberosidade fixa por parafusos.

A colocação da placa de ATT fixa por parafusos foi realizada na tíbia esquerda do mesmo cão doador. O procedimento é similar à colocação da placa convencional, com exceção da fixação na tuberosidade tibial, que é realizada por parafusos em vez do garfo. Marcação da osteotomia na tuberosidade tibial foi realizada com bisturi elétrico e osteotomia parcial da tuberosidade com serra oscilatória à bateria. Com $50 \%$ da osteotomia realizada, a placa foi fixa na região da tuberosidade com dois parafusos. Em seguida, a osteotomia foi completada. O espaçador foi posicionado na região proximal da osteotomia e fixo por meio de parafusos. Finalmente a placa foi fixa na região distal por dois parafusos de $2,7 \mathrm{~mm}$.

Foram realizados 34 ensaios piloto para adequação do dispositivo fixo à máquina e adequação da garra fixa ao ligamento patelar. Este foi um ensaio ex vivo em tíbias de cadáveres de cães submetidas a teste mecânico de tração axial. Os ensaios biomecânicos foram realizados utilizando-se máquina universal de ensaios mecânicos Kratos ${ }^{\circledR}$, modelo KE3000, dotada de célula de carga de $3000 \mathrm{~N}$. As tíbias em estudo foram submetidas a ensaio destrutivo até falha por soltura, quebra de implante ou quebra da crista, definido como ensaio de tração. O corpo de prova foi fixo ao dispositivo, e este fixo à máquina de ensaios por meio de garras circulares com parafusos. O joelho foi fixo em todos os ensaios em $135^{\circ}$ (Fig. 2) Uma terceira garra presa a quatro cabos de aço foi conectada à parte móvel da máquina de ensaios, e os cabos foram fixos ao ligamento patelar e submetidos à força de tração de $20 \mathrm{~mm} / \mathrm{min}$, transmitida da região da osteotomia da crista da tíbia até a falha 
(soltura/quebra dos implantes ou fratura da crista). Os parâmetros força e deformação foram enviados ao computador IBM $\AA$-PC por meio de um sistema de aquisição analógica TRACOMPW95 (TRCV61285), permitindo a gravação dos parâmetros e do gráfico para posterior análise. $\mathrm{O}$ mesmo procedimento foi realizado com o membro esquerdo do mesmo animal, cuja crista da tíbia foi fixa por placa e parafusos.

Os resultados foram avaliados comparativamente entre grupos para significância estatística, utilizando-se teste t-pareado. Significância estatística foi de $\mathrm{P} \leq 0,05$.

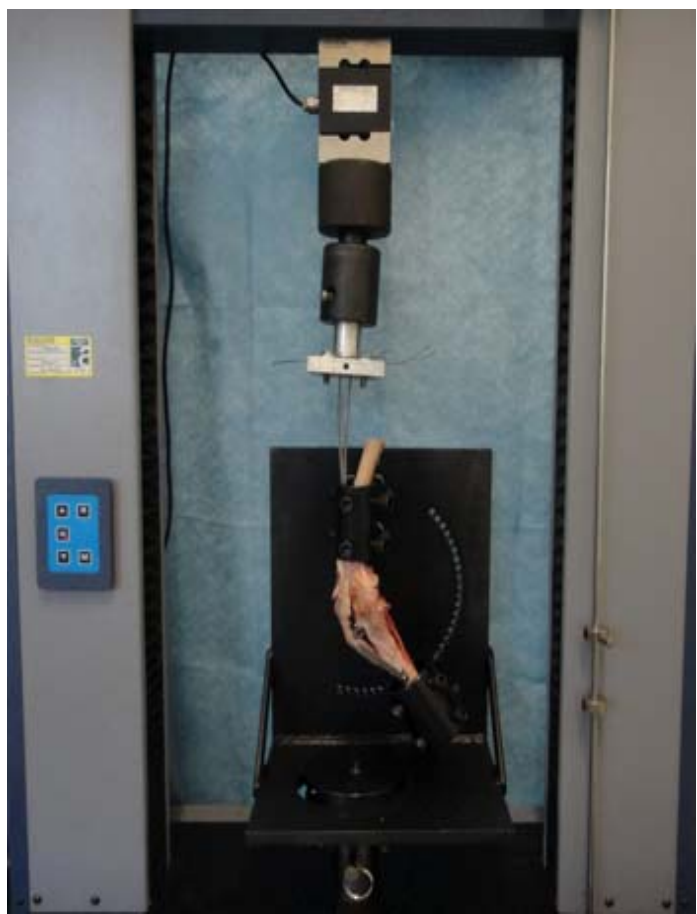

Figura 2. Demonstração do posicionamento do corpo de prova (fêmur e tíbia direitos do cão doador, submetido à técnica de ATT com placa convencional) no dispositivo preso à máquina de ensaios biomecânicos. O dispositivo mantém o corpo de prova na angulação anatômica do membro pélvico a $135^{\circ}$, mimetizando o apoio fisiológico. Dispositivo de tração fixo ao ligamento patelar por quatro cabos de aço conectados à célula de carga e à parte móvel da máquina de ensaios que aplicará a força de tração.
RESULTADOS

Todos os cães doadores dos membros pélvicos utilizados neste estudo atendiam critérios de inclusão propostos. Raça, sexo, peso e idade dos animais doadores dos membros e força máxima de tração aplicada no segmento tuberosidade/ligamento patelar das tíbias direita e esquerda em cada ensaio estão descritos na Tab. 1.

Para cada ensaio, o programa da máquina Kratos gerou um relatório de ensaio com gráfico e dado numérico de força máxima atingida até falha (fratura da tuberosidade tibial ou falha do implante), totalizando 20 ensaios, $10 \mathrm{em}$ cada grupo.

Entre as falhas observadas nos ensaios, nos membros pélvicos direitos, as tuberosidades fixas por garfo apresentaram dois casos de soltura do garfo, sete casos de fratura longitudinal da tuberosidade ao longo do garfo de fixação e um caso de fratura transversa da tuberosidade tibial na região proximal do garfo. Nas tuberosidades fixas por parafusos, observou-se, na região do parafuso proximal, quebra da cabeça do parafuso em três casos e soltura do parafuso em sete casos, fratura da crista na região do parafuso distal em sete casos e soltura do parafuso nos outros três casos. Em todos os ensaios, houve deslocamento proximal do espaçador, entretanto não houve soltura de seus parafusos de fixação.

Os resultados da carga máxima até a falha apresentaram distribuição normal, confirmada por meio do teste de Kolmogorov-Smirnov. Foi, então, utilizado o teste paramétrico t-pareado, com o objetivo de determinar se há diferença estatística entre os grupos (nível de significância de 0,05). As análises foram realizadas com o programa GraphPad InStart.

A média da carga máxima até a falha foi de $128,70 \mathrm{~kg} / \mathrm{F}$ (mínimo $104,55 \mathrm{~kg} / \mathrm{F}$ e máximo $151,80 \mathrm{~kg} / \mathrm{F}$ ) e de $141,99 \mathrm{~kg} / \mathrm{F}$ (mínimo $111,60 \mathrm{~kg} / \mathrm{F}$ e máximo $169,65 \mathrm{~kg} / \mathrm{F}$ ) no grupo utilizando garfo e parafusos, respectivamente. $\mathrm{O}$ desvio-padrão dos grupos garfo e parafuso foi baixo, 12,99 e 17,21, respectivamente. Foi encontrada diferença significativa $(\mathrm{P}=0,03)$ entre as médias dos grupos (Tab. 2). 
Tabela 1. Raça, sexo, peso e idade dos cães doadores dos membros pélvicos submetidos à técnica de ATT para confecção dos corpos de prova e força máxima de tração aplicada até a falha no segmento tuberosidade/ligamento patelar das tíbias direita (placa convencional de ATT fixa por garfo) e esquerda (placa de ATT fixa por parafusos) em cada ensaio

\begin{tabular}{|c|c|c|c|c|c|c|c|}
\hline & Raça & Sexo & $\begin{array}{l}\text { Peso } \\
(\mathrm{kg})\end{array}$ & Idade & $\begin{array}{l}\text { Resistên } \\
\text { máxima } \\
\text { tíbia } \\
\text { (garfo) }\end{array}$ & $\begin{array}{l}\text { ia } \\
(\mathrm{kg} / \mathrm{F}) \\
\text { direita }\end{array}$ & $\begin{array}{l}\text { Resistência } \\
\text { máxima }(\mathrm{kg} / \mathrm{F}) \\
\text { tíbia esquerda } \\
\text { (parafuso) }\end{array}$ \\
\hline 1 & Husky Siberiano & Fêmea & 20 & 7 anos & 104,55 & & 126,90 \\
\hline 2 & Pit Bull & Fêmea & 25 & 5 anos & 125,55 & & 169,65 \\
\hline 3 & Sem raça definida & Fêmea & 23 & 3 anos & 122,85 & & 131,10 \\
\hline 4 & Rottweiller & Macho & 30 & 5 anos & 128,85 & & 146,70 \\
\hline 5 & Pit Bull & Macho & 30 & 3 anos & 140,55 & & 160,80 \\
\hline 6 & Sem raça definida & Fêmea & 22 & 6 anos & 131,10 & & 147,75 \\
\hline 7 & Rottweiller & Fêmea & 25 & 2 anos & 136,95 & & 153,90 \\
\hline 8 & Pit Bull & Fêmea & 28 & 5 anos & 151,80 & & 134,70 \\
\hline 9 & Labrador & Fêmea & 30 & 7 anos & 128,10 & & 136,80 \\
\hline 10 & Sem raça definida & Macho & 20 & 5 anos & 116,70 & & 111,60 \\
\hline
\end{tabular}

Tabela 2. Número de amostras submetidas aos ensaios por grupo, força média, diferença entre médias, forças mínima e máxima registradas em cada grupo, desvio-padrão e valor de $\mathrm{p}$

\begin{tabular}{llc} 
& Grupo 1 (placa/garfo) & Grupo 2 (placa/parafuso) \\
\hline Número de amostras & 10 & 10 \\
Força média & $128,70 \mathrm{~kg} / \mathrm{F}$ & $141,99 \mathrm{~kg} / \mathrm{F}$ \\
Diferença entre médias & & $13,29 \mathrm{~kg} / \mathrm{F}$ \\
Força mínima & $104,55 \mathrm{~kg} / \mathrm{F}$ & $111,60 \mathrm{~kg} / \mathrm{F}$ \\
Força máxima & $151,80 \mathrm{~kg} / \mathrm{F}$ & $169,65 \mathrm{~kg} / \mathrm{F}$ \\
Desvio-padrão & 12,99 & 17,21 \\
Valor de p (teste t-pareado) & & 0,03 \\
\hline
\end{tabular}

\section{DISCUSSÃO}

Ensaios biomecânicos vêm sendo realizados com o intuito de quantificar resultados que, antes, hipotéticos, baseavam-se apenas em estudos clínicos (Boudrieau, 2005; Conzemius et al. 2005; Damur, 2005; Hoffmann et al., 2006; Baraúna et al., 2007; Stein e Schmoekel, 2008; Voss et al., 2008; Ferrigno et al., 2009; Guerrero et al., 2009; Dymond et al., 2010; Bisgard et al., 2011; Kemper et al., 2011; Steinberg et al., 2011). Entretanto, é sabido que a avaliação biomecânica pode trazer informações importantes para estudos clínicos futuros. Os principais estudos biomecânicos realizados até então buscam avaliar implantes e eficácia de técnicas cirúrgica para tratamento de ruptura do ligamento cruzado cranial (Romano et al., 2006; Apelt et al., 2007; Kim et al, 2009; Guerrero et al., 2009; Lins et al., 2009; Etchepareborde et al., 2010; Guerrero et al., 2011). A investigação da resistência da nova placa de ATT, fixa por parafusos na tuberosidade tibial, no presente trabalho, apresenta grande relevância e importância clínica, pois, por ter sido comprovada resistência superior à placa convencional de ATT, esta poderá ser utilizada com maior segurança, uma vez que apresenta inúmeros benefícios quando comparada à placa de ATT convencional. Esses benefícios foram observados por Bisgard et al. (2011), em trabalho clínico com esse modelo de placa. Neste trabalho, foram utilizados 10 pares de membros de cadáveres de cães, totalizando 20 peças, divididas em dois grupos de 10 peças cada. $\mathrm{O}$ número de 10 espécimes por grupo também foi escolhido com base em estudos biomecânicos similares (Apelt et al., 2007; Guerrero et al., 2009; Kim et al., 2009, Butler et al, 2011). Fêmur e tíbia foram colocados no dispositivo num ângulo fixo de $135^{\circ}$, com o objetivo de manter o membro na posição de apoio em estação, próxima do fisiológico no cão, e por ser o ângulo preconizado para imagem radiográfica de planejamento para cirurgia de ATT, corroborando outros autores em ensaios 
biomecânicos (Apelt et al., 2007; Guerrero et al., 2009; Butler et al., 2011). A velocidade de ensaio utilizada neste trabalho foi de $20 \mathrm{~mm} /$ minuto, baseada em ensaio biomecânico similar (Etchepareborde et al., 2010).

No presente experimento, a média da carga máxima até a falha foi de $128,70 \mathrm{~kg} / \mathrm{F}$ (mínimo $104,55 \mathrm{~kg} / \mathrm{F}$ e máximo $151,80 \mathrm{~kg} / \mathrm{F}$ ) e de $141,99 \mathrm{~kg} / \mathrm{F}$ (mínimo $111,60 \mathrm{~kg} / \mathrm{F}$ e máximo $169,65 \mathrm{~kg} / \mathrm{F})$ no grupo utilizando garfo e parafusos, respectivamente. Etchepareborde et al. (2010) realizaram ensaio biomecânico avaliando resistência à tração em osteotomias de tíbias utilizando técnica modificada semelhante à descrita por Maquet (1976) e observaram resultados de resistência máxima próximos aos obtidos neste ensaio nos diferentes grupos (média $128 \mathrm{~kg} / \mathrm{F}, 114 \mathrm{~kg} / \mathrm{F}$ e $62 \mathrm{~kg} / \mathrm{F}$ ).

No grupo tratado com placa de ATT convencional, a falha mais comum observada foi a quebra da crista tibial ao longo da inserção do garfo (7/10), falha comum observada também por outros autores em estudos clínicos anteriores (Hoffman et al., 2006; Lafaver et al., 2007; Stein e Schmoekel, 2008; Voss et al., 2008). É provável que a falha na região da tuberosidade tibial na região da inserção do garfo se dê por fragilidade causada pelos múltiplos orifícios do implante. Stein e Schmoekel (2008) alegam que a fratura na região da crista provocada por erro de técnica foi em razão da osteotomia da tuberosidade tibial estreita, o que não foi observado no presente trabalho. Outra falha observada no grupo tratado com placa convencional foi a soltura do garfo na região da tuberosidade tibial $(2 / 10)$. Acredita-se que isso ocorreu por impossibilidade de retorcimento da placa na região da tuberosidade. No grupo tratado com as placas de ATT fixas por parafusos, a falha mais comum observada foi fratura da tuberosidade tibial na inserção do parafuso distal (7/10), corroborando resultados clínicos observados por Bisgard et al. (2011), que observaram, entre complicações em seu relato, $75 \%$ dessa natureza.

Existem limitações a serem citadas neste estudo. Os testes foram realizados com carga continua e unidirecional e não puderam reproduzir todas as forças que atuam normalmente nos implantes em um joelho in vivo. Infelizmente, resistência mecânica in vivo não pode ser realizada em animais e humanos, por isso estudos ex vivo são realizados e podem fornecer informações úteis quanto à resistência de implantes em estudos experimentais biomecânicos controlados como este. Para o objetivo proposto pelo estudo, a carga contínua mostrou-se eficaz, e foram observadas semelhanças nas complicações apresentadas neste estudo e em estudos clínicos publicados (Bisgard et al., 2011). Ausência de musculatura adjacente também pode ser considerada como limitação do estudo, uma vez que a musculatura do quadríceps é extremamente importante na flexão e extensão do joelho, bem como na estabilidade articular e é necessária para restringir a translação cranial tibial (Nakamura et al., 1985). Ante essas limitações, houve o cuidado de se adequar e de se padronizar a maioria das variáveis nos grupos apresentados, a fim de serem obtidos resultados mais criteriosos.

O ensaio comparativo foi realizado entre membros pélvicos pertencentes ao mesmo cão doador, uma vez que, segundo autores (Budsberg et al., 1993; Gillette e Zebas, 1999), apesar de não existirem cães perfeitamente simétricos, comparando-se o movimento dos membros e os valores de pico vertical, não existe diferença significativa entre eles, o que reduz as variáveis entre os corpos de prova.

Avanço da tuberosidade tibial é um procedimento bem aceito como técnica para tratamento da ruptura do ligamento cruzado cranial (Hoffmann et al., 2006; Lafaver et al., 2007). Qualquer modificação nos implantes convencionais (placa de ATT e garfo de fixação) deve promover resistência similar ou maior aos implantes originais. Segundo os resultados, existe diferença significativa da placa fixa por parafusos na tuberosidade tibial quando comparada à placa convencional de ATT $(\mathrm{P}=0,03)$ em relação à resistência mecânica promovida em ensaio biomecânico ex vivo.

\section{CONCLUSÃO}

De acordo com resultados obtidos nos ensaios biomecânicos, placa de ATT fixa por parafusos na região da tuberosidade tibial promove resistência à tração significativamente maior quando comparada com placa fixa por garfo, que se dá por causar menor fragilidade óssea por menor número de orifícios para fixação na tuberosidade tibial. 


\section{AGRADECIMENTOS}

À Fundação de Amparo à Pesquisa do Estado de São Paulo (Fapesp), pela verba concedida para realização do experimento; ao Departamento de Cirurgia da Faculdade de Medicina Veterinária e Zootecnia da Universidade de São Paulo.

\section{REFERÊNCIAS}

APELT, D.; KOWALESKI, M. P.; BOUDRIEAU, R. J. Effect of tibial tuberosity advancement on cranial tibial subluxation in canine cranial cruciate deficient stifle joints: an in vitro experimental study. Vet. Surg., v.36, p.170-177, 2007.

BARAÚNA Jr., D.; ROEHSIG, C.; ROCHA, L.B. et al. Técnica de interligação extracapsular fêmoro-fabelo-tibial na ruptura do ligamento cruzado cranial em cães: achados clínicos e radiográficos. Ciênc. Rural, v.37, p.769-776, 2007.

BISGARD, S. K.; BARNHART, M. D.; SHIROMA, J. T. et al. The effect of cancellous autograft and novel plate design on radiographic healing and postoperative complications in tibial tuberosity advancement for cranial cruciate deficient canine stifles. Vet. Surg., v.40, p.402407, 2011.

BOUDRIEAU, R.J. Tibial tuberosity advancement (TTA): clinical results. In: ACVS VETERINARY SYMPOSIUM, 2005, San Diego. Proceedings... San Diego: American College of Veterinary Surgeons, 2005. p.27-29.

BUDSBERG, S.C.; EVENS, D.J.; BROWN, J. et al. Evaluation of limb symmetry índices, using ground reaction forces in healthy dogs. Am. J. Vet. Res., v.54. p.1569-1574, 1993.

BUTLER, J.R.; SYRCLE, J.A.; McLAUGHLIN, R.M. et al. The effect of tibial tuberosity advancement and meniscal release on kinematics of the cranial cruciate ligament-deficient stifle during early, middle, and late stance. Vet. Comp. Orthopaed. Traumatol., v.24, p.342-349, 2011.

CONZEMIUS, M.G.; EVANS, R.B.; BESANCON, M.F. et al. Effect of surgical technique on limb function after surgery of rupture of the cranial cruciate ligament in dogs. J. Am. Vet. Med. Assoc., v.226, p.232-236, 2005.
DAMUR, D. M. Tibial tuberosity advancement (TTA): clinical results. In: ACVS VETERINARY SYNPOSIUM, 2005, San Diego. Proceedings... San Diego: American College of Veterinary Surgeons, 2005. p.441442.

DYMOND, N.L.; GOLDSMID, S.E.; SIMPSON, S.J. Tibial tuberosity advancement in 92 canine stifles: initial results, clinical outcome and owner evaluation. Aust J., v.88, p.381-385, 2010

ETCHEPAREBORDE, S.; BRUNEL, L.; BOLLEN, G.; BALLIGAND, M. Preliminary experience of a modified Maquet technique for repair of cranial cruciate ligament rupture in dogs. Vet. Comp. Orthopaed. Traumatol., v.24, p.223-227, 2011.

FERRIGNO, C.R.A.; CUNHA, O.; MARIANI, T.C. et al. Tibial tuberosity advancement (TTA). O que é essa nova técnica para tratamento da ruptura do ligamento cruzado cranial em cães. Rev. Anclivepa, v.21, p.21-23, 2009.

GUERRERO, T.G.; MAKARA, M.A.; KATIOFSKY, K.K. et al. Comparison of healing of the osteotomy gap after tibial tuberosity advancement with and without use of an autogenous cancellous bone graft. Vet. Surg., v.40. p.27-33, 2009.

GUERRERO, T.G.; POZZI, A.; DUNBAR, N. et al. Effect of tibial tuberosity advancement on the contact mechanics and the alignment of the patellofemoral and femorotibial joints. Vet. Surg., v40, p.839-848, 2011.

GILlETTE, R.L.; ZEBAS, C.J. A twodimensional analysis of limb symmetry in the trot of Labrador retrievers. J. Am. Anim. Hosp. Assoc., v.35. p.515-520, 1999.

HOFFMANN, D.E.; MILLER, J.M.; OBER, C.P. et al. Tibial tuberosity advancement in 65 canine stifles. Vet. Comp. Orthopaed.Traumat., v.19, p.219-227, 2006.

JUNG, H.J.; VANGIPURAM, G.; FISHER, M.B. et al. The effect of multiple freeze-thaw cycles on the biomechanical properties of the human bone-patellar tendon-bone allograft. $J$. Orthop. Res., v.29, p.1193-1198, 2011. 
KEMPER, M.; KOCH, D.; BASS, M.; INAUEN, R. Major complication rate after 214 tibial tuberosity advancements as a therapy for cranial cruciate ligament rupture. In: ORTHOPEDICS SIVEMAP (SIMPOZIJUM VETERINARA MALE PRAKSE SRBIJE), 2011, Belgrado Sérvia. Proceedings... Belgrado, Sérvia: SIVEMAP, 2011. p.1-3.

KIM, S. E.; POZZI, A.; BANKS, S.A. et al. Effect of tibial tuberosity advancement on femorotibial contact mechanics and stifle kinematics. Vet. Surg., v.38, p.33-39, 2009.

KIM, S.E.; POZZI, A.; KOWALESKI, M.P.; LEWIS, D.D. Tibial osteotomies for cranial cruciate ligament insufficiency in dogs. Vet. Surg., v.37, p.111-125, 2008.

LAFAVER, S.; MILLER, N.A.; STUBBS, W.P. et al. Tibial tuberosity advancement for stabilization of the canine cranial cruciate ligament- deficient stifle joint: surgical technique, early results and complications in 101 dogs. Vet. Surg., v.36, p.573-586, 2007

LINS, B.T.; RAHAL, S.C.; LOUZADA, M.J. et al. Mechanical resistance of the modified stabilization method for the tibial tuberosity advancement technique. Ex vivo experimental study in dogs. Ciênc. Rural, v.39, p.467-472, 2009.

MAQUET, P. Advancement of the tibial tuberosity. Clin. Orthop. Relat. Res., v.115, p.225-230, 1976.

MONTAVON, P. M.; DAMUR, D. M.; TEPIC, S. Advancement of the tuberosity for the treatment of cranial deficient stifle. In: WORLD ORTHOPAEDIC VETERINARY CONGRESS, 1., 2002, Munich, Germany. Proceedings... Munich, Germany: ESVOT, 2002. p.152.
NAKAMURA, N.; ELLIS, M.; SEEDHOM, B.B. Advancement of the tibial tuberosity - a biomechanical study. J. Bone Joint Surg., v.67, p.255-260, 1985.

ROMANO, L.; PEREIRA, C.A.M.; SCHMAEDECKE, A. et al. Análise biomecânica do joelho íntegro e com ruptura do ligamento cruzado cranial quanto ao grau de deslocamento cranial e rigidez articular em cães. Acta Cir. Bras., v.21, p.47-51, 2006.

SLOCUM, B., DEVINE, T. Cranial tibial thrust: a primary force in the canine stifle. J. Am. Vet. Med. Assoc., v 183, p.456-459, 1983.

STEIN, S.; SCHMOEKEL, H. Short-term and eight to 12 months results of a tibial tuberosity advancement as treatment of canine cranial cruciate ligament damage. J. Small Anim. Pract., v.49, p.398-404, 2008

STEINBERG, E.J.; PRATA, R.G.; PALAZZINI, K.; BROWN, D.C. Tibial tuberosity advancement for treatment of $\mathrm{CrCL}$ injury: complications and owner satisfaction. J. Am. Anim. Hosp. Assoc., v.47. p.250-257, 2011.

VOSS, K.; DAMUR, D.M.; GUERRERO, T. et al. Force plate gait analysis to assess limb function after tibial tuberosity advancement in dogs with cranial cruciate ligament disease. Vet. Comp. Orthop. Traumatol., v.21, p.243-249, 2008.

WOLF, R.E.; SCAVELLI, T.D.; HOELZLER, M.G. et al. Surgical and postoperative complications associated with tibial tuberosity advancement for cranial cruciate ligament rupture in dogs: 458 cases (2007-2009). J. Am. Vet. Med. Assoc., v.240, p.1481-1487, 2012. 